\title{
The mechanical response of semiflexible networks to localized perturbations
}

\author{
D.A. Head ${ }^{1,2}$, A.J. Levine ${ }^{3,4}$ and F.C. MacKintosh ${ }^{1}$ \\ ${ }^{1}$ Department of Physics and Astronomy, Vrije Universiteit, Amsterdam, The Netherlands. \\ ${ }^{2}$ Department of Applied Physics, The University of Tokyo, Tokyo 113-8656, Japan. \\ ${ }^{3}$ Department of Physics, University of Massachusetts, \\ Amherst MA 01060, USA. ${ }^{4}$ Department of Chemistry $\&$ Biochemistry, \\ University of California, Los Angeles, CA 90095
}

(Dated: October 2, 2018)

\begin{abstract}
Previous research on semiflexible polymers including cytoskeletal networks in cells has suggested the existence of distinct regimes of elastic response, in which the strain field is either uniform (affine) or non-uniform (non-affine) under external stress. Associated with these regimes, it has been further suggested that a new fundamental length scale emerges, which characterizes the scale for the crossover from non-affine to affine deformations. Here, we extend these studies by probing the response to localized forces and force dipoles. We show that the previously identified nonaffinity length [D.A. Head et al. PRE 68, 061907 (2003).] controls the mesoscopic response to point forces and the crossover to continuum elastic behavior at large distances.

PACS numbers: 87.16.Ka, 82.35.Pq, 62.20.Dc
\end{abstract}

\section{INTRODUCTION}

Semiflexible polymers such as filamentous proteins resemble elastic rods on a molecular scale, while exhibiting significant thermal fluctuations on the scale of micrometers or even less. This has made them useful as model systems allowing for direct visualization via optical microscopy. But, semiflexible polymers are not just large versions of their more well-studied flexible cousins such as polystyrene. Filamentous proteins, in particular, have been shown to exhibit qualitatively different behavior in their networks and solutions. A fundamental reason for this is the fact that the thermal persistence length, a measure of filament stiffness as the length at which thermal bending fluctuations become apparent, can become large compared with other important length scales such as the spacing between polymers in solutions, or the distance between chemical crosslinks in a network.

One of the most studied semiflexible polymers in recent years has been F-actin, a filamentous protein that plays a key structural role in cells [1, 2, 3]. These occur in combination with a wide range of specific proteins for crosslinking, bundling and force generation in cells. These composites, together with other filamentous proteins such as microtubules, constitute the so-called $c y$ toskeleton that gives cells both mechanical integrity and structure. This biopolymer gel is but one example of a large class of polymeric materials that can store elastic energy in a combination of bending and extensional deformations of the constituent elements. Such systems can be called semiflexible gels or networks.

One of the important lessons from recent experimental and theoretical studies is that the shear modulus of cross-linked semiflexible networks bears a much more complex relationship to the mechanical properties of the constituent filaments and to the microstructure of the gel than is the case for flexible polymer gels [4]. Recently, it has been shown that semiflexible gels exhibit a striking cross-over 5, 6, 7, 8, 9] between a response to external shear stress that is characterized by a spatially heterogeneous strain (a non-affine regime [10,11]) and a uniform strain response (an affine regime 12]). This crossover is governed primarily by cross-link density and molecular weight (filament length). The bulk shear modulus of the network simultaneously increases by about six orders of magnitude at this nonaffine-to-affine cross-over. The underlying mechanism responsible for this abrupt cross-over appears to be the introduction of a new, mesoscopic length scale in the problem that is related to both the bending stiffness of the constituent polymers and the mean spacing between consecutive cross-links along the chain [5, 8].

One can associate this mesoscopic length with the length below which the deformation of the network departs from the standard affinity. The nonaffinity length $\lambda$, introduced in Refs. [5, 8], can be qualitatively understood as the typical length over which one finds nonaffine deformation in the network. In this previous work we presented a scaling analysis that relates this mesoscopic length scale to the network density and the stretching and bending moduli of the constituent filaments. The macroscopic shear response of the network is then controlled by a competition between $\lambda$ (the nonaffinity length) and the filament length, $L$. On the one hand, when the filament length is long, nonaffine corrections to the deformation field, which are localized to regions within $\lambda$ of the filament ends, do not significantly affect the mechanical properties of the network; the shear modulus of the macroscopic system is well-described by calculations based on affine deformation reflecting the fact that nonaffine deformations of the filament ends are subdominant corrections in this limit. Moreover, the elastic energy is stored primarily in the (homogeneous) extension and compression of filaments. On the other hand, when the filaments are of a length comparable to, or shorter than the nonaffinity length, i.e. $L \lesssim \lambda$ then the nonaffine de- 
formations of the ends play a large, even dominant role in determining the mechanical properties of the network. The network is found to be generally more compliant, and the elastic energy under applied shear stress is stored in a spatially heterogeneous manner in the bending of filaments. The existence of these distinct regimes as a function of filament length reflects a fundamental difference of these semiflexible polymer networks with respect to their flexible counterparts: polymers can maintain their mechanical integrity and state of stress/strain across network nodes or crosslinks.

These results naturally lead one to pose a number of basic questions regarding the elastic properties of semiflexible networks. While these random networks must on basic theoretical grounds appear as continuum, isotropic materials at the longest length scales, these considerations do not predict the length at which the continuum approximation applies. The previous identification of the nonaffinity length, $\lambda$ as the only mesoscopic length associated with the nonaffine-to-affine cross-over in uniformly sheared semiflexible networks suggests that this length should more generally control the cross-over to continuum behavior [8]. After all the affine deformation of the network under uniform stress at scales large compared to $\lambda$ shows that in one case at least the nonaffinity length controls the cross-over to continuum behavior. One of the principal results of the present work is the demonstration that $\lambda$ more generally controls this cross-over to continuum mechanics in semiflexible gel systems.

Prior work has focussed exclusively on simple shear and uniaxial extension. In order to better examine the universality of the previous results, we study the opposite limit of a highly localized external force in the form of a point force monopole or dipole. If one can show that the elastic (displacement) Green's function of the system similarly depends on only one additional parameter $\lambda$ then it would appear that this quantity completes the coarse-grained elastic description of the system on all length scales down to the mean distance between crosslinks. It may be, however, that the deformation field of these semiflexible networks is much more complex and the simplification introduced by $\lambda$ in the description of the network's response to uniform shear strain cannot be generalized to deformations resulting from more general forcing conditions.

While our results below, indeed, show that $\lambda$ does largely control the crossover to (the far field) continuum elasticity, the observed elastic Green's function is sensitive to the local structure of the network on length scales below $\lambda$. Below we discuss how we quantify the structure of the Green's function and its approach to the form required by continuum elasticity. The observed elastic Green's function, however, depends not only on the $\lambda$-dependent Lamé coefficients of the material, but also on local properties of the displacement field immediately surrounding the the point force. In effect one may imagine that, upon the application of the point force, the network acts as a type of composite material: within a distance $\lambda$ of the point force it deforms in a way not well described by continuum theories, while outside of that zone it does appear to act like an elastic continuum. The complete Green's function depends, of course, on the material properties of both media. Unfortunately, only one of those media (the outer zone) is well characterized by the simple continuum theory of an isotropic elastic solid, so the complete Green's function remains complex and depends on the detailed network structure within the inner zone surrounding the applied force.

There is another set of questions that may be addressed via the study of the network's response to point forces and force dipoles. Such forces not only probe the material properties of the network in a manner complimentary to the uniform strains explored earlier, they also have direct physical implications for microrheology in F-actin networks and for the dynamics of the cytoskeleton in response to the activity of nanoscale molecular motors, e.g. myosin. Fluctuation-based microrheology, an application of the fluctuation-dissipation theorem to the study of rheology via the statistical analysis of the thermally fluctuating position of sub-micron tracer particles embedded in the medium, requires one to understand the elastic Green's function of the medium. Thus understanding the response of semiflexible networks to localized forces has direct experimental implications and consequences for force production in the cytoskeleton.

In the biological context, the semiflexible network making up the cytoskeleton is generally found in association with molecular motors that, to a good approximation, generate transient localized force dipoles in the material. To both understand force generation in the cell as well as the material properties of these cytoskeletal networks driven into nonequilibrium steady states by these molecular motors, one must determine the displacement field associated with such motor-induced forces.

Notwithstanding our biological motivation for this work, our findings also bear on the broader problem of elastic modes in amorphous materials. It has been shown that the vibrational modes of deep-quenched LennardJones systems approach a continuum description only on scales exceeding some mesoscopic length $\xi$ [13, 14]; for the protocols considered, a value $\xi \sim 30$ particle dimensions was robustly found. This was physically identified with a length scale for non-affinity, suggesting a direct correspondence with our $\lambda$ (although our $\lambda$ can be controlled by varying the mechanical properties of the constituents). A comparable length was also found to control the self-averaging of the Green's function to the form expected by continuum elasticity [15]. These findings for radially interacting particles are broadly in keeping with our own investigations for semiflexible polymer networks. We also mention here that the relationship between continuum elasticity and the Green's function has also been discussed for mildly disordered spring networks [16].

The remainder of this paper is organized as follows: In section [11 we develop our model of semiflexible, permanently cross-linked gels, summarize the numerical simula- 
tions used to study it, and discuss the expected structure of the displacement field when averaged over numerous realizations of the network. In section III we report our results for both point forces in $\amalg I \mathrm{~A}$ and force dipoles in IIIB We then discuss our studies of the bulk elastic properties of these networks in 1 C before concluding in section IV

\section{MODEL}

\section{A. The semiflexible network}

A highly successful continuum continuum model of individual semiflexible polymers is the worm-like chain. This treats the linear filaments as elastic rods of fixed contour length and negligible thickness, so that the dominant contribution to the elastic energy comes from bending modes and the Hamiltonian linearized to small deviations from a straight configuration that is given by

$$
\mathcal{H}^{\perp}=\frac{1}{2} \kappa \int\left(\nabla^{2} u\right)^{2} \mathrm{~d} s,
$$

where $u$ is the transverse displacement of the filament relative to an arbitrary straight axis, $s$ is its arc length, and the elastic modulus $\kappa$ gives the bending energy per unit length $\delta s$.

The longitudinal response of the wormlike chain model is calculated from the increase in free energy due to an extensional stress applied along the mean filament axis [12, 17]. However, the numerical algorithm used in our simulations is based on the minimization of the Hamiltonian of the system, and hence is fundamentally athermal. The reason for this choice is essentially one of efficiency: assuming there are no subtle convergence issues, minimization is expected to be faster than stochastic modelling and, it is anticipated, give better statistics for a given CPU time. This does, however, mean that the entropic mechanism governing the longitudinal response is absent, and an explicit energetic term is required.

An unconstrained filament at $T=0$ forms a straight configuration, and thus elongation of its end-to-end distance must be accompanied by a change in the absolute contour length. It is therefore natural to incorporate longitudinal modes by adding a second elastic term to the Hamiltonian for the extension or shortening of the filament backbone,

$$
\mathcal{H}^{\|}=\frac{1}{2} \mu \int\left(\frac{\mathrm{d} l(s)}{\mathrm{d} s}\right)^{2} \mathrm{~d} s,
$$

where $d l(s) / d s$ gives the strain or relative change in local contour length, and $\mu$ is the Young's modulus of the filament (essentially a spring constant normalized to $1 /$ [length]). Of course, such modes also exist in thermal systems, but may be dominated by the entropic spring terms [12], except possibly for very short filament segments or very densely cross-linked gels. The connec- tion between thermal/entropic and athermal longitudinal compliance is discussed in greater detail in Ref. [8].

The two elastic coefficients $\kappa$ and $\mu$ together define a length scale $l_{\mathrm{b}}=\sqrt{\kappa / \mu}$, which will shall refer to as the intrinsic bending length by observing that an isolated filament constrained to have different tangents at its end points will deform with this characteristic length. To avoid potential confusion, however, we note that this is not the typical length scale for bending deformations of a semiflexible filament. Rather, the bending energy of filaments tends to make the longest unconstrained wavelength bending mode the dominant one. Thus, for instance, in a crosslinked gel, filaments are expected to be bent primarily on a length comparable to the distance between crosslinks. Nonetheless $l_{\mathrm{b}}$ is a useful measure of filament rigidity, in that large $l_{\mathrm{b}}$ corresponds to rigid filaments, and small $l_{\mathrm{b}}$ to flexible ones.

Although $\kappa$ and $\mu$ have been introduced as fundamental coefficients, if the filament is regarded as a continuous elastic body with uniform cross section at zero temperature, then they can both be expressed in terms of the characteristic filament radius $a$ and intrinsic bulk modulus $Y_{\mathrm{f}}$ as $\kappa \sim Y_{\mathrm{f}} a^{4}$ and $\mu \sim Y_{\mathrm{f}} a^{2}$. Thus $l_{\mathrm{b}} \sim a$, and thinner filaments are more flexible than thick ones (as measured by $l_{\mathrm{b}}$ ), as intuitively expected. Given the possibility of entropic effects in $\mu$, however, we shall treat these as independent parameters of the theory [8]

The gel is constructed by depositing filaments of monodisperse length $L$ and zero thickness onto a twodimensional substrate. The center-of-mass position vector and orientation of the filaments are uniformly distributed over the maximum allowed range, so the system is macroscopically isotropic and homogeneous. Whenever two filaments overlap they are cross-linked at that point. Deposition continues until the required mass density, as measured by the mean distance between crosslinks $l_{\mathrm{c}}$, has been reached. The network thus constructed can be described by three lengths: $L, l_{\mathrm{b}}$, and $l_{\mathrm{c}}$ and one modulus scale: $\mu$. In two-dimensions $l_{\mathrm{c}}$ characterizes both the mass density and cross-link density in spatially random, isotropic networks. The length $l_{\mathrm{b}}$ characterizes the mechanical properties of the constituent filaments via the ratio of their bending to stretching compliance. The overall modulus scale $\mu$ will be absorbed into the point forces applied to the network.

Previously [5, 8], we identified an additional length $\lambda=l_{\mathrm{c}}\left(l_{\mathrm{c}} / l_{\mathrm{b}}\right)^{z}$, where $z \simeq 1 / 3$. This non-affinity length characterized the crossover from non-affine to affine network response. Specifically, for filament lengths $L$ much larger than $\lambda$ (i.e., high molecular weight), the bulk network properties could be understood quantitatively in terms of affine strains, while significant non-affine effects were observed for $L \lesssim \lambda$. Although this length arose naturally from considerations of bulk network properties [5], its dependence on $l_{\mathrm{c}}$ and $l_{\mathrm{b}}$ can be understood in terms of a balance of stretching/compression and bending energies of a single filament, treated in a self-consistent way within a network 8 . It is important to note that this 
(material) length is intermediate, between the (geometric) network length $l_{\mathrm{c}}$ and the macroscopic scale. In fact, in dilute networks, for which $l_{\mathrm{c}} \gg l_{\mathrm{b}}$, we shall argue below that the network can be thought of on scales $\gtrsim \lambda$ as a quasi-continuum: continuous, as opposed to discrete, but not necessarily described by macroscopic continuum elasticity. We shall find that this non-affinity length will play a key role in our analysis of the displacement field on this intermediate/quasi-continuum scale.

In order to apply the point forces, a crosslink is chosen at random and identified as the origin of the system; it is this crosslink that will later be perturbed. A fixed circular boundary at radius $R$ from the origin is imposed, and any filaments or filament segments that extend beyond the boundary are simply removed or truncated, respectively. Filaments ending on the rigid boundary are fixed there by another freely rotating bond as are found at all cross-links in the system. The allowed free rotation at the boundary means that the boundary supplies arbitrary constraint forces on the network but cannot support any localized torques.

\section{B. Numerical method}

Details of the simulation method have been presented elsewhere [8]. Here we briefly summarize the procedure, with particular attention on those aspects that are central to the problems studied in this paper.

The system Hamiltonian $\mathcal{H}\left(\left\{\mathbf{x}_{\mathbf{i}}\right\}\right)$ is constructed from discrete versions of (11) and (2) applied to the geometry generated by the random deposition procedure described above. The degrees of freedom $\left\{\mathbf{x}_{\mathbf{i}}\right\}$, or 'nodes,' are the position vectors of both crosslinks and midpoints between crosslinks, the latter so as to incorporate the first bending mode along the filament segments. Additional nodes could be included at the cost of additional run time, but are expected to have a small effect, since the longest unconstrained wavelengths tend to dominate bending deformations. Crosslinks are treated as constraints on the relative position of each connected filament segment, but not on their relative rotation. Physically this corresponds to an inextensible but freely rotating linkage. As previously noted, constrained bending at crosslinks has a small effect except at high network concentrations (specifically, when $l_{\mathrm{c}}$ becomes comparable to $a$ ) [8]. Nodes on the boundary are immobile. Note that, as in our earlier work [5, 7, 8], the network is assumed to be initially unstressed on both macroscopic and microscopic length scales.

There are two ways in which the system may be perturbed. The first, which we call monopole forcing, is to apply an arbitrarily small external force $\delta \mathbf{f}^{\text {ext }}$ to the crosslink at the origin. The network is then allowed to relax to a new configuration consistent with mechanical force balance at every node. The second, which we call dipole forcing, is to introduce a geometrical defect into the system by moving the central crosslink along the con- tour length of one of the filaments to which it belongs, but not the other. Physically, this corresponds, e.g., to a motor introducing relative motion of one filament with respect to another filament. In the simulations, this effect is incorporated by infinitesimally 'shifting' the image of the central node with respect to other nodes on one filament.

Once the perturbation has been specified, the displacements of the nodes in the new mechanical equilibrium are calculated by minimizing the system Hamiltonian $\mathcal{H}\left(\left\{\mathbf{x}_{\mathbf{i}}\right\}\right)$ using the conjugate gradient method [18]. This generates a displacement field for the particular geometry under consideration, as shown in Fig. 11 Note that $\mathcal{H}\left(\left\{\mathbf{x}_{\mathbf{i}}\right\}\right)$ is linearized about small nodal displacements $\left\{\delta \mathbf{x}_{i}\right\}$ from their original positions $\left\{\mathbf{x}_{i}\right\}$, so linear response is assured. The bulk response to non-linear strains has been recently studied by Onck et al. [19].

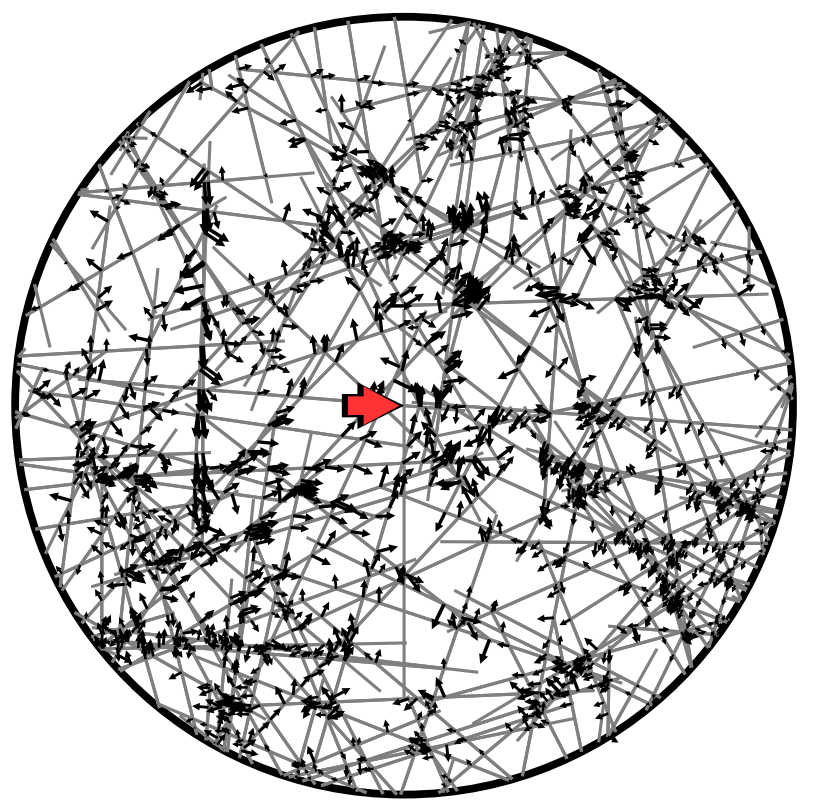

FIG. 1: (Color online) An example of a network of filaments of uniform length $L$ (grey line segments) perturbed by an external force, denoted by the large red arrow, applied to the crosslink at the origin of a circular system of radius $R=L$. The arrow lengths are logarithmically calibrated to the magnitude of the displacement of at each crosslink. In this example, $L / l_{\mathrm{c}} \approx 29.1, \lambda / L \approx 0.191$ and the force is perpendicular to one of the filaments that form the central crosslink; forces can also be applied parallel to a filament.

\section{Decomposition of mean displacement field}

As shown in Fig. 11 the displacement field for a particular network is quite complex and generically shows anticorrelations between displacements and the local mass distribution. Although these fluctuations reflect inherent and possibly interesting physical properties of the gels, a more basic and immediately applicable quantity 
to measure is the mean displacement field, found by averaging many individual runs with differing geometries but identical system parameters $R, L, l_{\mathrm{c}}$ and $\lambda$ (or equivalently $R, L, l_{\mathrm{c}}$ and $l_{\mathrm{b}}$ ). Two examples for differing $\lambda$ (the radius of the green circle centered on the point of force application) are given in Fig. 22 These plots demonstrate one of the primary results of this paper, namely that the crossover between continuum (or quasi-continuum) response at large lengths, to a more exotic displacement field at shorter lengths, happens at a length of order $(\lambda)$ with a prefactor close to unity.

In order to precisely describe the structure of the deformation field, we consider its most general possible form. For monopole forcing, the displacement field $\mathbf{u}(\mathbf{r})$ at position vector $\mathbf{r}$ relative to the origin can be projected onto 3 other vectors, namely the direction of the external force $\hat{\mathbf{f}}$, the unit position vector $\hat{\mathbf{r}}$ and the axis $\hat{\mathbf{n}}$ of one of the filaments to which the crosslink is attached,

$$
\begin{aligned}
u_{i} & =G_{i j} \hat{f}_{j}, \\
G_{i j} & =\frac{f}{\mu_{\text {aff }}}\left\{g^{(r)} \hat{r}_{i} \hat{r}_{j}+g^{(n)} \hat{n}_{i} \hat{n}_{j}+g^{(f)} \delta_{i j}\right\}
\end{aligned}
$$

where $f$ is the magnitude of the external force, and $\mu_{\text {aff }}$ is the shear modulus as predicted for affine deformation. This depends on $L$ and $l_{\mathrm{c}}$ but not $\lambda$, and is included here to factor out the density dependence of the response. As defined, the $g^{(\cdot)}$ (and the $h^{(\cdot)}$ below) are dimensionless quantities in two dimensions.

Each $g^{(\cdot)}$ can be further decomposed into angular modes in $\theta$, where $\cos \theta=\hat{\mathbf{n}} \cdot \hat{\mathbf{r}}$,

$$
g^{(\cdot)}=g_{0}^{(\cdot)}+2 \sum_{\substack{m>0, m \text { even }}} g_{m}^{(\cdot)} \cos (m \theta) .
$$

Terms in $\sin (m \theta)$ vanish since the ensemble-averaged response must be invariant under $\theta \leftrightarrow-\theta$, and $\cos (m \theta)$ terms with $m$ odd also vanish due to $\hat{\mathbf{n}} \leftrightarrow-\hat{\mathbf{n}}$ invariance. This latter symmetry may appear to violate the known polarity of typical semiflexible biopolymers such as F-actin and microtubules [20]; however, we are interested here in the mechanical properties of the filaments, which, within the approximations of our model, are indeed invariant under $\hat{\mathbf{n}} \leftrightarrow-\hat{\mathbf{n}}$.

For dipole forcing, the above procedure is followed with the additional simplification that $\hat{\mathbf{n}}$ is proportional to $\hat{\mathbf{f}}$, since the displacement (and hence force) dipole induced by the motion of a motor will always be parallel to one filament axis. The decomposition is therefore somewhat simpler,

$$
\begin{aligned}
u_{i} & =H_{i j} \hat{n}_{j}, \\
H_{i j} & =\frac{f}{\mu_{\text {aff }}}\left\{h^{(r)} \hat{r}_{i} \hat{r}_{j}+h^{(n)} \delta_{i j}\right\} .
\end{aligned}
$$

The scalar $f$ is the magnitude of the force dipole; since it is actually a displacement that is imposed, $f$ is unknown as will be treated as a fitting parameter. The angular
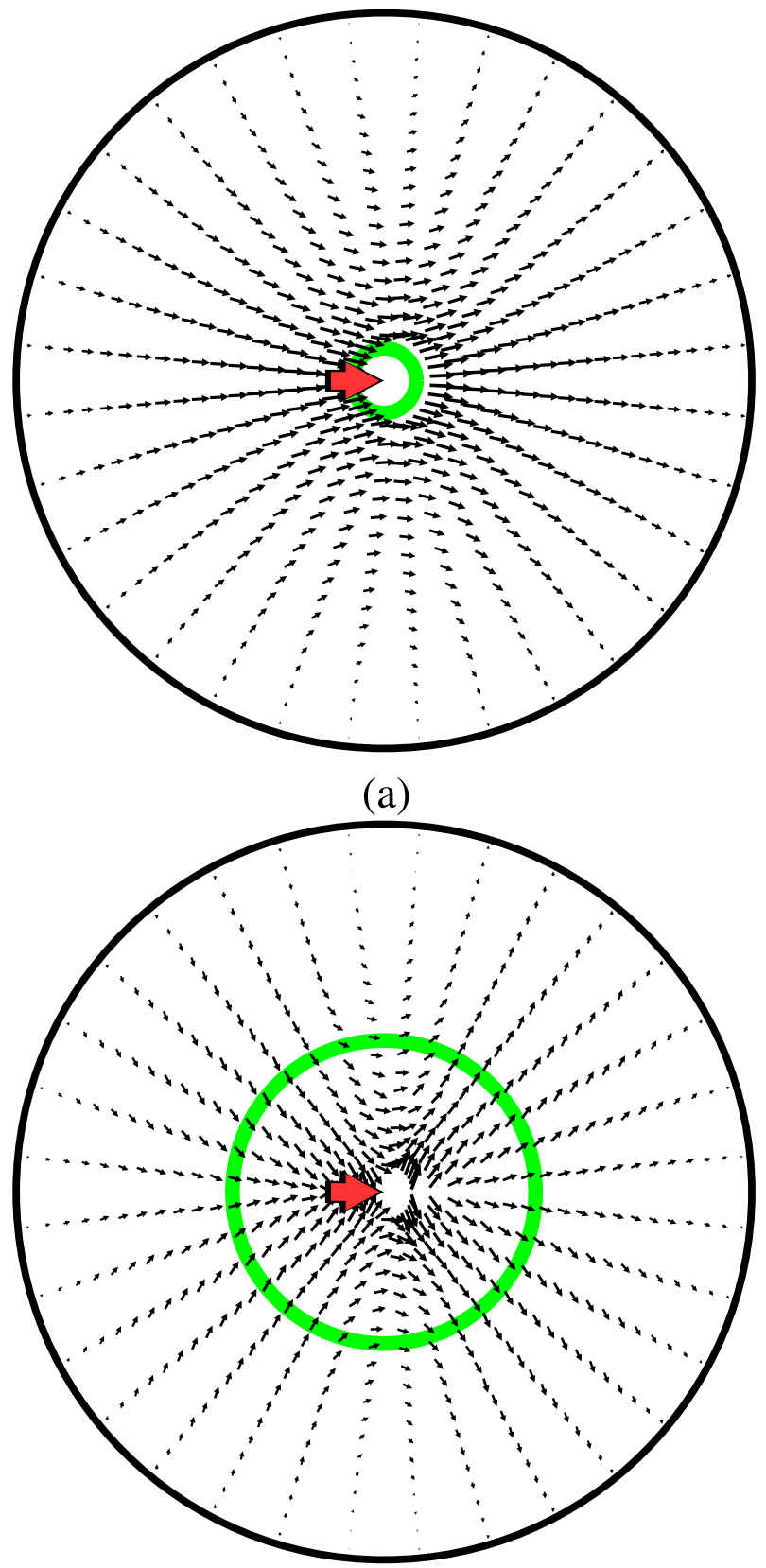

(b)

FIG. 2: (Color online) Mean response after averaging $\mathcal{O}\left(10^{5}\right)$ networks with the same parameters as in Fig. (1) (including one filament fixed perpendicular to the external force) except for $\lambda$, which is (a) $\lambda / L \approx 0.089$ and $(b) \lambda / L \approx 0.42$. For easy visualization, a green circle of radius $\lambda$ has been inserted into the background of each plot. Vectors near the center of each system have not been plotted for clarity.

decomposition is identical to before,

$$
h^{(\cdot)}=h_{0}^{(\cdot)}+2 \sum_{\substack{m>0, m \text { even }}} h_{m}^{(\cdot)} \cos (m \theta) .
$$

Later sections will refer to the continuum solution for 
each of the two forms of forcing. These are given in the appendix. For the monopole case, only two continuum modes are non-zero, namely $g_{0}^{(r)}$ and $g_{0}^{(f)}$. We shall refer to these components of the Green's function as continuum modes in order to distinguish from those components (non-continuum modes) that must vanish in the continuum. We do this even though for our finite systems the non-continuum modes do not vanish.

The dipole modes are slightly more subtle: after averaging of many dipole fields generated by the simulation, the resulting field is quadrupolar. This is an immediate consequence of the means of forcing the system. Recall that in an elementary step, a motor moves parallel to one filament axis. This has the effect of compressing the filament in front of the dipole, while stretching the trailing segment. Thus two filament segments are perturbed, each of which can be treated as a force dipole which, for a particular network geometry, will be of different magnitudes and hence the resulting field is dipole. However, the net bias of this dipole is symmetrically distributed around zero, and thus vanishes after averaging, leaving a quadrupole field as shown in Fig. 3. As derived in the appendix, the non-zero modes for this field are $h_{0}^{(r)}, h_{2}^{(r)}$, $h_{0}^{(f)}$ and $h_{2}^{(f)}$.

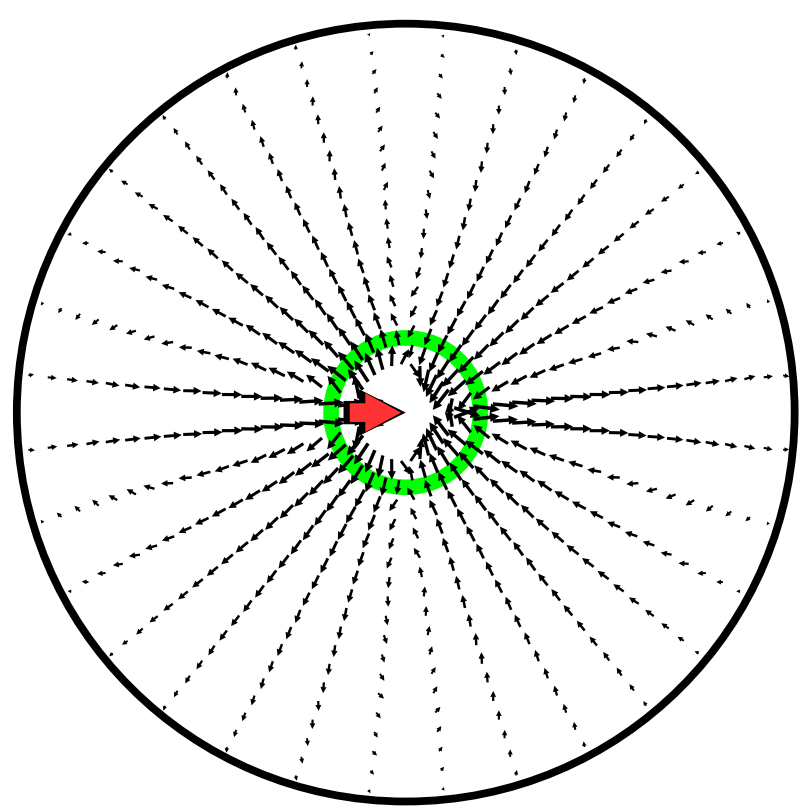

FIG. 3: (Color online) Mean displacement field after averaging over $\mathcal{O}\left(10^{5}\right)$ individual fields induced by imposing a displacement dipole at the origin. The orientation of the dipole is given by the red arrow. As explained in the text, the mean dipole moment vanishes after averaging and the resulting field is quadrupole. A green circle at a radius $\lambda=0.191 L$ from the origin is also shown.

\section{RESULTS}

The mechanical response to a localized perturbation depends on the distance from the point of perturbation. We divide the discussion of these results into the following parts. First, we examine the response to a point force using the decomposition of the displacement field outlined in previous section. We contrast the decay of the non-continuum modes of the displacement field with the behavior of the continuum modes of the displacement field and discuss the finite-size effects in the simulations. At large length scales, we find that the deformation field approaches a quasi-continuum form, in which all but a small number of ensemble-average modes decay rapidly toward zero. The remaining modes of the deformation field are the same as those predicted by continuum elasticity. In other words, the strain field about a point force reflects the expected tensorial character and rotational symmetries based on continuum elasticity theory. We find that $\lambda$ again plays a central role in controlling the cross-over from the near-field to the quasi-continuum.

We then turn to the spatial structure of the elastic energy density field around the point force paying particular attention to the partitioning of that energy density between stretching and bending modes of the filaments. We observe that the ratio of these energy contributions achieves the bulk value over much shorter distances from the point force than does the structure of the displacement field acquire its far-field, or bulk structure. We then extend our analysis to consider force dipoles in the medium. Lastly, we extend our previous analysis of the bulk elasticity of the filament network by examining both the Young's modulus and the Poisson ratio of the network.

\section{A. The response to point forces}

\section{The Displacement Field}

In this section we focus on the short length scale behavior of the monopole response, for which the noncontinuum modes are non-zero. We wish to distinguish two distinct forms of this non-continuum behavior: (i) higher angular modes $g_{m}^{(r)}$ and $g_{m}^{(f)}$ with $m>0$ are non-zero, and (ii) the $g_{m}^{(n)}$ modes do not vanish, i.e. the response depends on the orientation of the filament to which the force is applied. This latter observation gives a clear indication of how the response can 'see' the microscopic structure of the gel on short length scales.

An example demonstrating the appearance of noncontinuum modes at short lengths is given in Fig. [4 which shows the $g_{2}^{(f)}$ mode for systems with different crosslink densities $L / l_{\mathrm{c}}$ but with the filament flexibility chosen to give the same $\lambda \approx 0.191 L$ in each case. This plot shows the decay of the $\cos (2 \theta)$ amplitude of the component of the displacement field in the direction of the 
applied force (at the origin). In all three systems one observes a rapid decay of this angular harmonic that must vanish for a continuum isotropic system. Moreover, the characteristic length scale for this decay appears to be of order $\lambda(\simeq 0.2 R)$, although we examine this point more quantitatively below. For all network densities and values of $\lambda$ studied it is clear that the magnitude of $g_{2}^{(f)}$ vanishes rapidly with distance from the point force. No data are shown for larger values of $L / l_{\mathrm{c}}$ since at high network densities the numerical convergence of the strain field is so slow as to prevent attaining meaningful statistics.

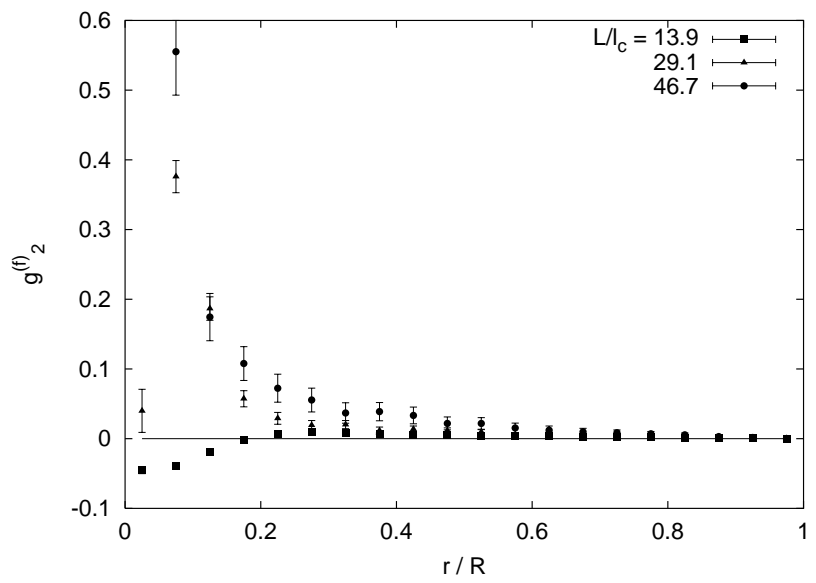

FIG. 4: $g_{2}^{(f)}$ (which is dimensionless) versus distance from point of force application $r$ for systems with the same $\lambda / L \approx$ 0.191 and crosslink densities $L / l_{\mathrm{c}}$ as given in the key ( $L$ is the filament length). The system radius $R=L$ in all cases.

This picture of non-continuum modes decaying to near zero at a length comparable to $\lambda$ holds also for the $m>2$ modes of $g_{m}^{(f)}$ and for all the $m>0$ modes of $g_{m}^{(r)}$. For reasons of space we do not show these data here. Instead we present data in Fig. 5 that demonstrate the decay of $g_{0}^{(n)}$ with $r$ for three networks densities such that in each case $\lambda \approx 0.191 L$. This non-continuum mode of the displacement field measures the circularly averaged amplitude of the component of displacement in the direction $\hat{n}$, i.e. along the axis of the rod to which the force has been applied. Clearly, such behavior has no counterpart in an isotropic continuum elastic model. It is interesting to note that this amplitude also appears to decay exponentially with a decay length of order $\lambda$.

In order to test quantitatively whether $\lambda$ indeed controls the decay of those components of the displacement field that have no counterparts in the continuum theory, we examine, as an example, $g_{2}^{(f)}$ vs. $r$ more closely in Fig. 6] Here we plot this for a range of values of networks density and of $\lambda$. If, as suggested above, the decay is exponential with characteristic length $\lambda$, then plotting these data log-linear with radial distances scaled by $\lambda$ should cause all these curves to exhibit the same slope. We have shifted the data sets vertically in order to facilitate visual comparison. (We note that, although a

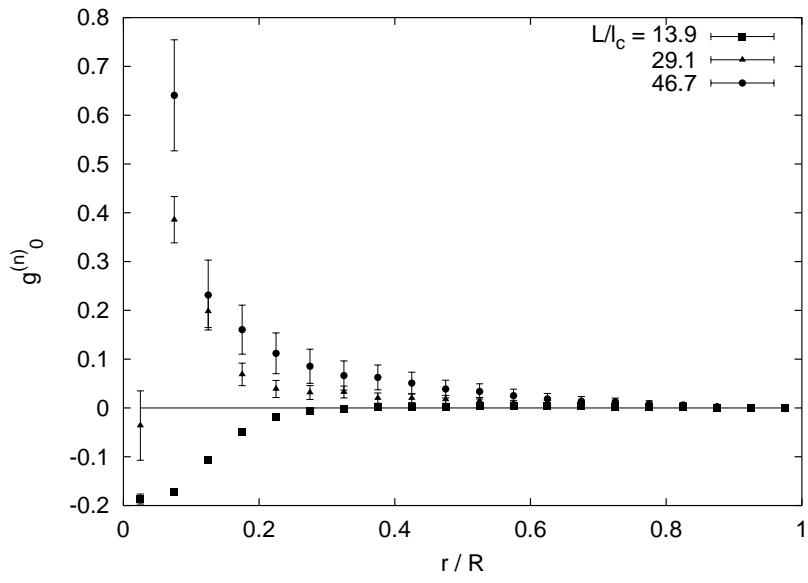

FIG. 5: $g_{0}^{(n)}$ versus $r / R$ for $\lambda / L \approx 0.191, R=L$ and the $L / l_{\mathrm{c}}$ given in the key.

known force is applied to the origin of our sample, since we expect the unknown local constitutive relations in our system to depend on density and other parameters, the amplitudes cannot be directly compared. Here, we wish only to establish the nature of the decay of the noncontinuum modes.) We have also introduced the solid lines corresponding to $\exp (-r / \lambda)$ merely as guides to the eye. These are not fits, although fits to these data for various $L / l_{c}$ and $\lambda$ demonstrate decay lengths of $\lambda$ within $10 \%$.

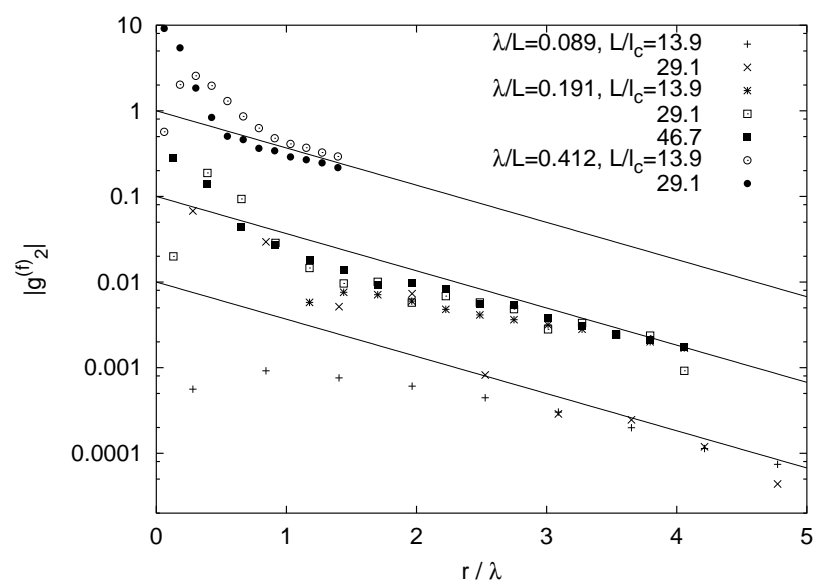

FIG. 6: Log linear plots of $g_{2}^{(f)}$ versus the scaled distance from perturbation $r / \lambda$ for systems with various values of $\lambda$ as given in the key. The system radius $R=L$ in all cases. Data within a boundary layer of width $\lambda$ from $r=R$ is not shown (see text for details).

Thus, we observe a near field regime in which the decay of the $g_{2}^{(f)}$ appears to be generically more rapid than $\exp (-r / \lambda)$ followed by the quasi-continuum regime where the exponential decay with decay length $\lambda$ is observed. This demonstrates that the nonaffinity length $\lambda$, indeed, controls the approach toward the expected con- 
tinuum behavior (i.e., vanishing of the non-continuum modes). Similar results (not presented here for reasons of space) are also found for the other non-continuum modes. Specifically, the decay lengths are also found to be $\lambda$ within $10 \%$.

Of course, the fixed zero-displacement boundary condition at $r=R$ requires all components of the displacement field to vanish there. The essential distinction between the continuum and non-continuum modes lies in the manner in which their amplitudes decay upon approach to the rigid boundary. The non-continuum modes examined above decay exponentially with a decay length proportional to $\lambda$. We demonstrate in the next section that the continuum mode amplitudes, in contrast, do not appear to decay in the same way. In particular, their approach to zero at the rigid boundary is not controlled by $\lambda$.

\section{Continuum modes and finite-size effects}

In this section we address two related points: (i) the fundamental change in the structure of the displacement field as one moves away from the immediate vicinity of the applied force, and (ii) the role of finite size effects in our numerical simulation in two-dimensions. We cannot address the former point without confronting the latter one for the following reason. Due to the presence of the rigid boundary, all modes of the displacement field decay to zero at the boundary.

We show in this section, however, that the decay of the non-continuum modes is controlled by internal, mesoscopic length scales in the network, while the decay of the continuum modes is determined by the macroscopic geometry of the system including the presence of the rigid boundary. Since the distinction between the continuum and non-continuum modes depends essentially on the presence of the boundary and specifically on the separation of the system size $R$ from the internal length scales controlling the decay of the non-continuum modes $(\propto \lambda)$, we consider this distinction along with a more general discussion of finite size effects in our simulation.

To have a well-defined elastic response in twodimensions (meaning that the displacement field vanish at large distances from the applied point force) one needs to impose a rigid boundary. In order to eliminate the differential influence of the boundary on the various angular modes of the displacement field we chose this boundary to be a circle of radius $R$ centered on the point of force application. This rigid boundary forces all components of the displacement field to vanish exactly at $r=R$. Such a rigid boundary can be expected to introduce a boundary layer near the edges of our system, which we observe and discuss below.

Figure 17 shows the decay of the $g_{0}^{(r)}$ mode amplitude for systems of size $R=L$ and $\lambda \approx 0.191 L$. We note that the decay of this continuum mode is qualitatively distinct from the decay of the non-continuum modes shown

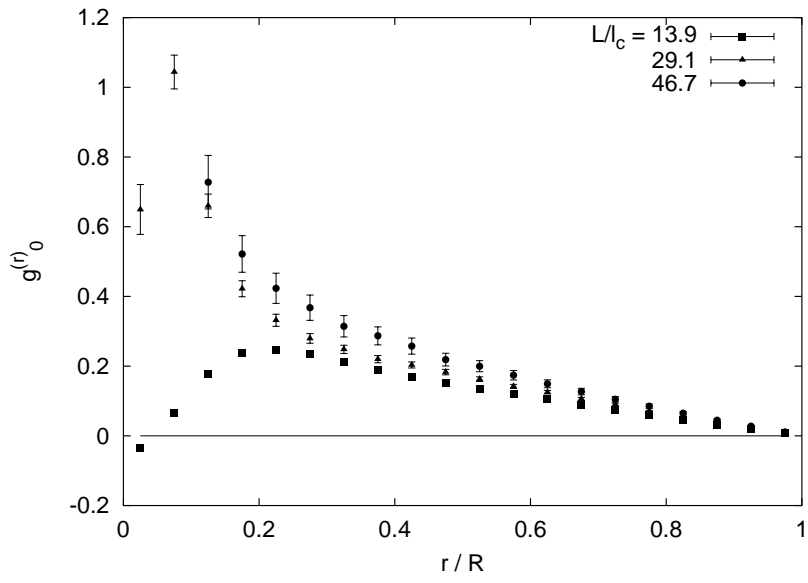

FIG. 7: $g_{0}^{(r)}$ versus $r / R$ for $\lambda / L \approx 0.191, R=L$ and differing $L / l_{\mathrm{c}}$ as shown in the legend.

in Figs. 45 and 6 We do not observe an exponential decay of this continuum mode amplitude. Nevertheless, observing an exponential decay having a long decay length, or, more reasonably, the product of an algebraic function and such a weak exponential decay would be difficult to resolve in this plot.

To study these issues further, we plot in Fig. $8 g_{0}^{(r)}$ for various system sizes from $R=L / 2$ to $5 L$. We observe both an apparent convergence for the larger systems to a common curve when distances are measured relative to the size of the system, as well as a systematic down-turn near the boundary. The first of these observations suggests that the sample geometry controls the dependence of this continuum mode, as opposed to intrinsic lengths like $\lambda$. The down-turn in these data within a distance of order $\lambda$ (here, $\simeq 0.2 L$ ) of the boundary is to be expected from the observations above concerning the role of the length scale $\lambda$. One can view this crossover length as the distance along a filament over which a force applied to the filament dissipates/expands into a (quasi-)continuum stress/displacement field. Thus, the effects of the boundaries on the individual filaments contacting the boundary are expected to propagate at least this distance into the system. Because of this, we have also removed all data within a distance $\lambda$ of the boundaries in Fig. 6 above, which exhibit a similar down-turn near the boundary.

In Fig. 9 we examine the radial dependence of $g_{0}^{(r)}$ for three different values of $\lambda / L$ for a large system where $R=3 L$. In this case, we see a coincidence of the data for different $\lambda$, indicating that the long-range behavior of $g_{0}^{(r)}$ is not controlled by $\lambda$.

Although we were unable to obtain sufficient statistics on the non-continuum (and sub-dominant) modes for larger systems, the combination of the qualitatively different behavior from the continuum modes, together with the consistent dependence on the crossover length $\lambda$ lead us to conclude that the principal results of the prior section are not strongly influenced by finite-size effects. 
Thus, $\lambda$ controls the disappearance of the non-continuum modes of the system.

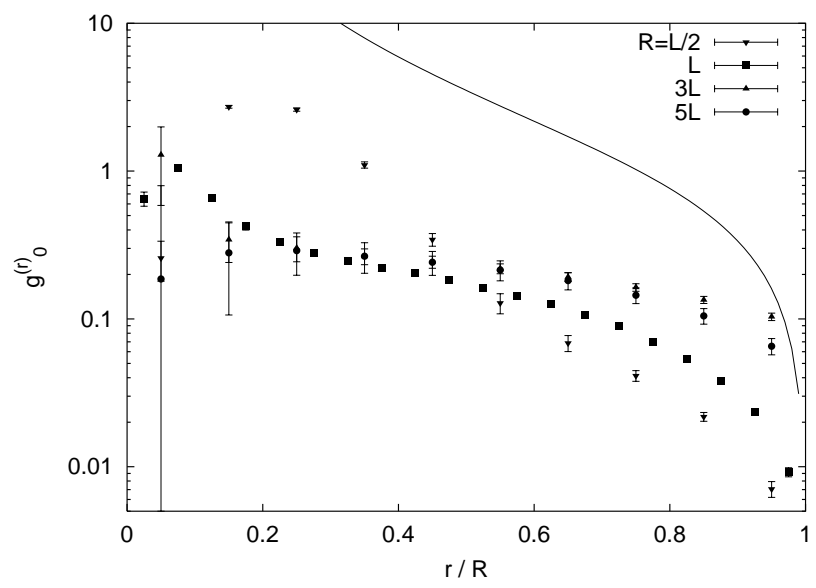

FIG. 8: $g_{0}^{(r)}$ versus $r / R$ for $\lambda / L \approx 0.191, L / l_{\mathrm{c}} \approx 29.1$ and the system radii $R$ given in the key. The continuum response for the bulk elastic moduli corresponding to these parameter values (as given by (9) is shown as a solid line.

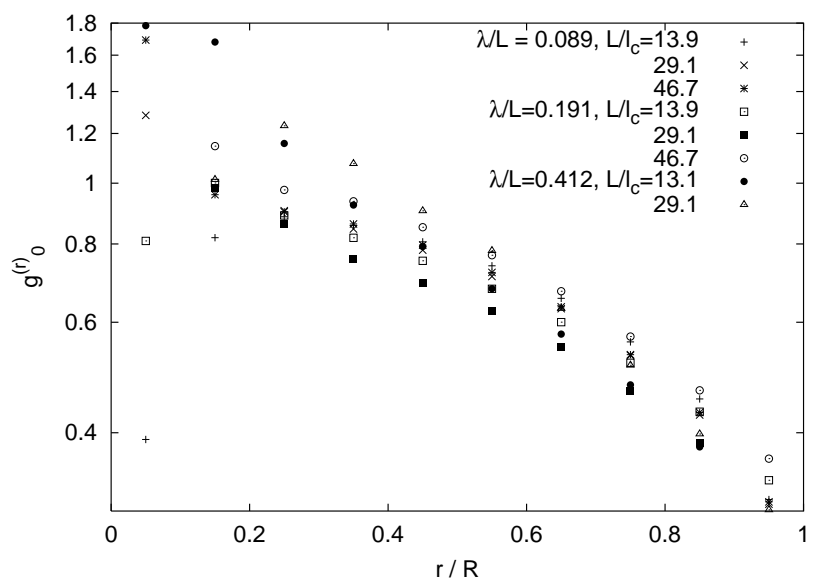

FIG. 9: $g_{0}^{(r)}$ versus $r / R$ for $R=3 L$ on $\log$-linear axes, with $\lambda / L$ and $L / l_{\mathrm{c}}$ as given in the key. Each curve has been shifted vertically by a $\lambda$-dependent scale factor to attempt data collapse. Since $\lambda$ varies by over a factor of 4 , the rough collapse is enough to rule out any significant $\lambda$-dependence on the decay. Error bars are not shown for clarity.

We also see a large discrepancy between the expected continuum solution and the observed displacement of the simulated filament network as shown in Fig. 8 This difference cannot be simply attributed to finite-size effects; as can be seen in Fig. 8 there is no observable convergence of the larger system data for the continuum modes to the predictions of continuum elasticity for a material having the appropriate Lamé coefficients. Nevertheless, we clearly observe the more rapid decay of the non-continuum modes on a length-scale controlled by $\lambda$. One is left with the following puzzle: The tensorial structure and the rotational symmetry of each component of the displacement field approaches the form required by the continuum theory, but the continuum model appears to never quantitatively agree with the numerical data.

We may speculate about the underlying cause of this discrepancy. It is clear that that the deformation of the material in the immediate vicinity of the point force (this "near zone" extends out to a distance $\propto \lambda$ from the point force) and in a boundary region at the rigid wall is not well described by any continuum theory. We suggest that under the application of the point force at the origin, the network effectively partitions itself into three different elastic materials. In the near field region $r<\lambda$ around the point force the deformation response to the point force is quite complex. Similar complexities appear to exist near the rigid wall, where $R-r<\lambda$. These regions apply a complex set of tractions on the circles $r \approx \lambda$ and $r \approx R-r$ bounding the intermediate region that deforms in a manner consistent with some continuum elastic material. Because these tractions are not themselves determined by a simple, continuum model, the resultant deformation of the intermediate region is also not simply derivable from an analysis of the elastic Green's function for a continuum.

One might imagine that for very large systems having a consequently larger intermediate region, the complexities of the tractions in the transition zones become less significant. Because of the rigid boundary at $r=R$ and the low-dimensionality of the system, this may not be the case. The continuum Green's function contains logarithmic terms and, due to the rigid boundary, growing polynomial terms as well. Thus, we do not expect convergence to the continuum Green's function even for significantly larger systems. One may ask whether three dimensional systems will show similarly poor convergence to the continuum solutions. Further research here is needed.

We suggest that we do indeed observe the approach of the structure of the elastic Green's function to that predicted by continuum theory. We term the region surrounding the point force where the deformation field has the expected form the quasi-continuum. Based on our numerical data, we do not expect to find a region in which the deformation field agrees quantitatively with the predictions of continuum elasticity using the Lamé coefficients appropriate to the medium as determined by uniform stress measurements. This suggests that semiflexible networks admit a highly complex point force response that cannot be fully captured by continuum elasticity even in the far-field. The full implications of this complexity have not been explored.

\section{Energy Density}

Another instructive measure of the monopole response is the density of elastic energy $\rho_{\mathrm{E}}$ at a given distance from the point perturbation, which measures gradients of the displacement field and therefore complements the analysis of $\mathbf{u}(\mathbf{x})$ given above. In addition, since the simulation 
contains explicit terms for the transverse and longitudinal filament deformation modes, it is straightforward to measure the partitioning of the energy between these modes. Based on previous work [5, 6, [] ], under homogeneous shear strain the partitioning of elastic energy between the bending and stretching modes of the filaments is determined entirely by the affine-to-nonaffine crossover. The ratio of $L / \lambda$ was found to control this energy partitioning at a macroscopic or average level. It remains to be seen how this partitioning of the elastic energy occurs in vicinity of a point force.

The freedom to choose the angle between the point force and the direction of the filament to which that force is applied allows one to determine locally the partitioning of the elastic energy between bending and stretching modes of the filaments. Forces directed along the filament axis generate primarily stretching deformations in the immediate vicinity of the origin (where the force is applied). Forces directed perpendicular to the filament axis, however, locally create a large bending deformation. As seen in the previous homogenously imposed strain deformation calculations, for any given value of $L / \lambda$ the network responds very differently to the bending or stretching deformations. Thus it is not surprising that the decay of the energy density from the point of force application to the boundary is strongly dependent on whether the force is applied parallel or perpendicular to one of the filaments at the crosslink.

Fig. 10 shows $\rho_{\mathrm{E}}$ for parallel forces, where $\lambda$ is fixed but the filament density $L / l_{\mathrm{c}}$ varies. There is an approximate data collapse onto a single curve as shown. The most notable discrepancy occurs near the boundary, where the low-density data remains higher than those for larger $L / l_{\mathrm{c}}$ values. This is most likely due to the boundary layer already discussed above for the displacement modes.

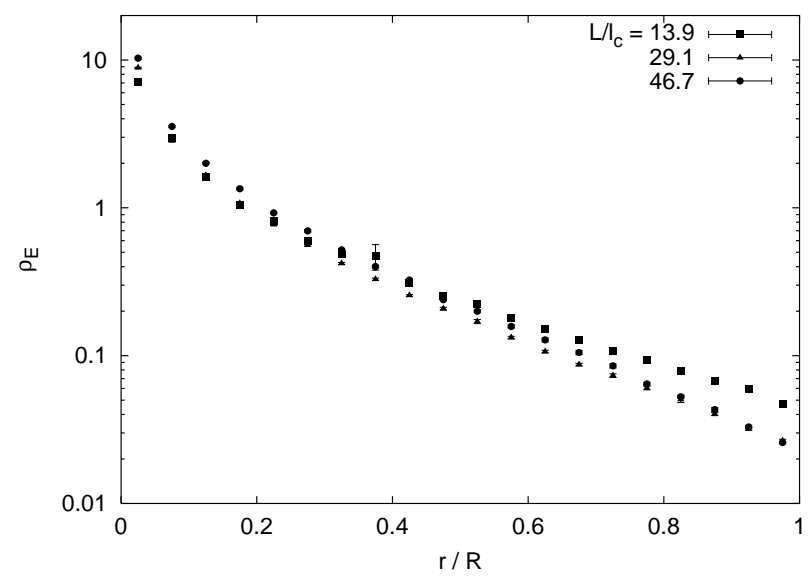

FIG. 10: Density of elastic energy $\rho_{\mathrm{E}}$ (in arbitrary units) versus distance $r$ from an externally applied force that is directed along the filament to which it is applied. $L / l_{\mathrm{c}}$ is varied as given in the key, and $R=L$ and $\lambda / L \approx 0.191$ in all cases.

In contrast, $\rho_{\mathrm{E}}$ for forces perpendicular to a filament exhibits much richer behavior, as shown in Fig.11] which
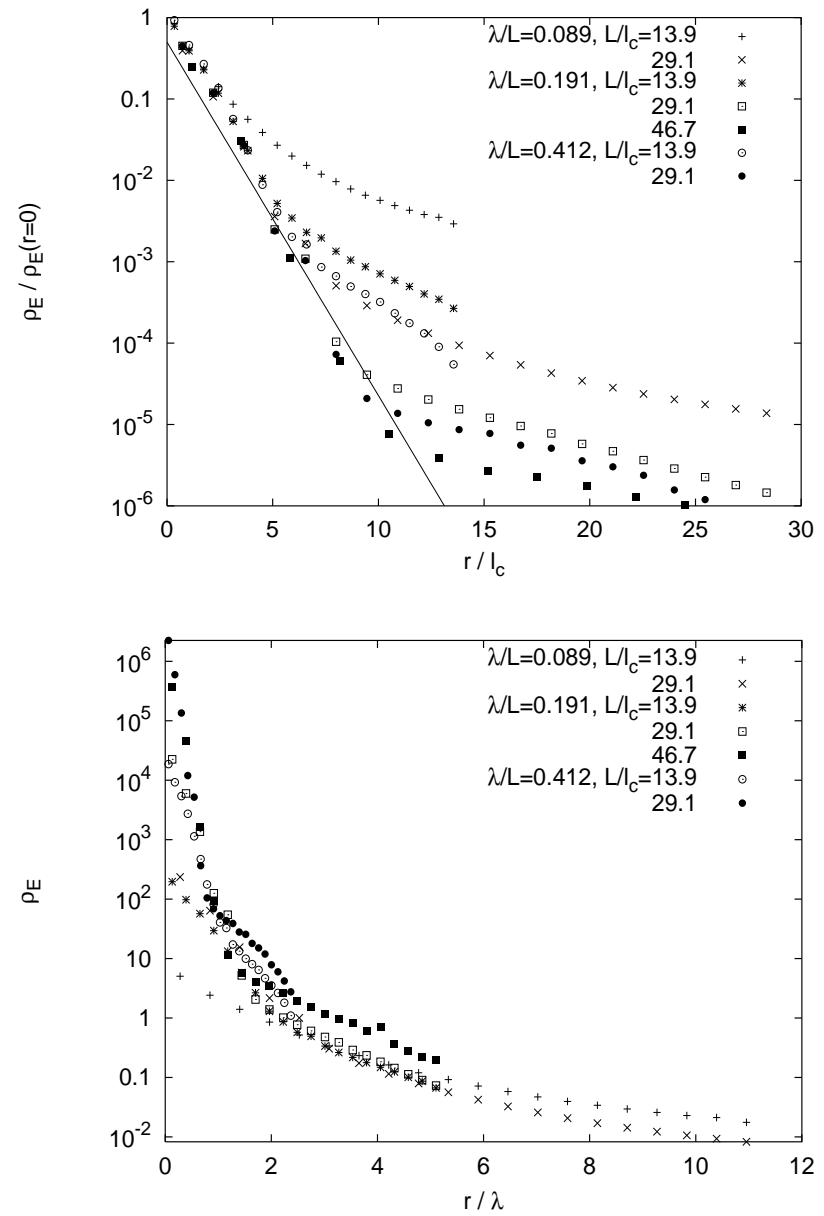

FIG. 11: Scaled elastic energy density versus distance from the point force for a variety of networks - see legend. In each case the force is directed perpendicularly to the filament where it is applied. In the upper panel distances are scaled by $l_{\mathrm{c}}$ and the data has been collapsed in the small $r / l_{\mathrm{c}}$ regime. The solid line is proportional to $\exp \left(-r / l_{\mathrm{c}}\right)$, showing $l_{\mathrm{c}}$ controls the initial decay of elastic energy. In the lower panel the same energy densities are plotted against distance scaled by $\lambda$. This shows the energy density decays with that characteristic length scale at longer ranges. Error bars are not shown for reasons of clarity.

shows the same data plotted against $r / l_{\mathrm{c}}$ and $r / \lambda$. For large distances, the data for different $L / l_{\mathrm{c}}$ appear to differ by only a scale factor. Since there is one arbitrary multiplicative factor for each curve (as only the magnitude of the applied force is fixed, not its displacement and hence work done on the network), these portions of the curves can be made to collapse after scaling, as in the parallel force case, suggesting that beyond some near-field regime, the decay of elastic energy density is once again exponential and governed by $\lambda$ - see Fig. 11 lower panel. However, in this near-field regime for perpendicular forces there is clearly no possibility of such a collapse. Instead, it appears that the more rapid decay of elastic energy density in this near-field regime is gov- 
erned by the length $l_{c}$, as can be seen in the upper panel of Fig. 11] where the (rescaled) energy density data are plotted vs. $r / l_{\mathrm{c}}$ and shown to decay as $\sim \exp \left(-r / l_{\mathrm{c}}\right)$ for distances $r \lesssim 5 l_{\text {c }}$ (ignoring finite size effects).

Based on the two different regimes of data collapse shown in Fig. 11] we conclude that there is a near-field region in which the decay of elastic energy is governed by a microscopic length scale-the mean distance between cross-links-and a longer-range regime in which the spatial decay of elastic energy density is controlled by the mesoscopic length $\lambda$. To better study this cross-over, we examine the partitioning of elastic energy between bending and stretching modes of the filaments. Recall that in the affine limit, the elastic energy is stored primarily in the stretching and compression of the filaments. In the nonaffine regime, on the other hand, the elastic energy is stored almost entirely in bending modes of the filaments.

Plotted in Fig. 12 is the proportion of elastic energy due to longitudinal filament deformation for the perpendicular force case. Three data sets are displayed having different values of $l_{c}$ and $\lambda$-see figure caption. The distance from the point of force application has been scaled by the geometric mean of $l_{c}$ and $L$ leading to the observed coincidence of the crossover between regimes in the data. Close to the point of the application of the force, the displacement response is clearly dominated by bending modes of the filaments. This is to be expected since the perpendicularly directed force directly injects bending energy into the system at the origin - the point of force application. The energy partitioning is inconsistent with that which is expected based on previous homogeneous shear measurements. From that work, one expects the network to determine the energy partitioning based only on the ratio: $L / \lambda$. We observe the fraction of stretching energy to rapidly increase towards the expected value based on $L / \lambda[\underline{5},[8]$ and we may characterize each curve has having a 'knee' separating the region of varying energy ratio from a nearly constant intermediate regime where the partitioning of elastic energies corresponds well with the previously identified fraction of stretch/compression energy under macroscopic strain. This correspondance is demonstrated in Fig. 13. The horizontal lines show the fraction of stretching energy in periodic systems subjected to macroscopic shear, and clearly coincide with the plateau reached beyond the knee. At the largest distances one notices the vanishing of bending energy as the fraction of stretching energy approaches unity. This last effect is due to the fact that each filament has a freely rotating bond at the outer, rigid wall. As this wall cannot support torques, the bending energy vanishes in a boundary layer whose width is determined by the mean distance between cross-links. We return to this point below.

The observed coincidence of the knees of all three curves under the rescaling of distances by $\sqrt{l_{c} L}$ suggests that this length sets the scale over which injected bending energy is redistributed into the combination of bending and stretching appropriate for long-length scale deforma-

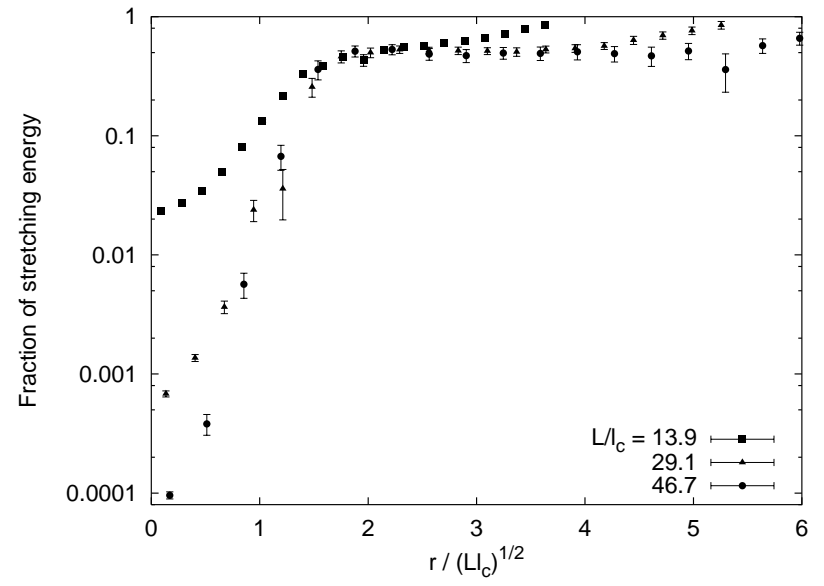

FIG. 12: Proportion of elastic energy due to filament stretching, $\mathcal{H}^{\|} /\left(\mathcal{H}^{\|}+\mathcal{H}^{\perp}\right)$, for three systems having differing values of $l_{c}$ (see key) but the same value of $\lambda$.

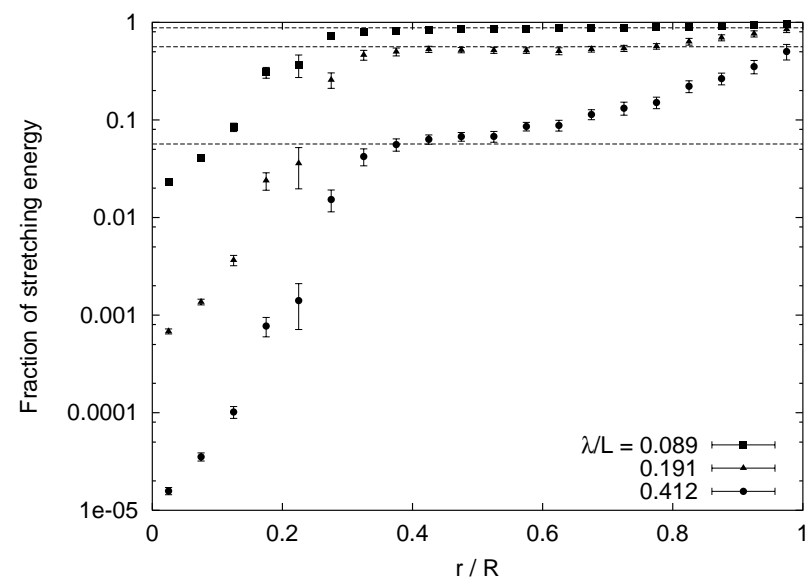

FIG. 13: The fraction of elastic energy due to filament stretching in networks with the same $L / l_{\mathrm{c}} \approx 29.1$ (in fact, the exact same geometries), $R=L$ but different $\lambda$. The different values of the stretching energy fraction in the intermediate regime reflect the differing values of the ratio $L / \lambda$ for the networks. For comparison, the macroscopic energy fraction under bulk shear is shown as horizontal dashed lines, in the same order as the data points (i.e. $\lambda / L \approx 0.089,0.191$ and 0.412 from top to bottom).

tions.

Based on these observations we note that in all networks the strain field acquires the structure of the point force response based on continuum elasticity over a (typically mesoscopic) length scale of $\lambda$. When the networks is subjected to large, local bending deformations, however, it readjusts the partitioning of bending to stretching energy over a generally much shorter length scale, $l_{c}$. Thus the system is able to repartition the local elastic energy storage to the value appropriate for its $L / \lambda$ ratio over smaller length scales than does the system recover the expected long length scale structure of its continuum 
elastic response.

We have already shown that in the intermediate field regime the fraction of stretching elastic energy in the system approaches its far-field value as determined by the ratio: $L / \lambda$. Nevertheless, we in fact find that at the edges of our sample the network energy becomes stored solely in stretching modes regardless of the value of $L / \lambda$. As mentioned above we attribute this final redistribution of the elastic energy density between bending and stretching modes to a boundary effect imposed by the freelyrotating nature of coupling of the network filaments to the rigid boundary. To further test that this final redistribution is indeed a boundary effect, we consider a few larger system sizes as shown in Fig. 14 In that figure the force remains perpendicular to the direction of the filament to which it is applied while the system size is varied from $R=L$ to $R=5 L$ for a network of fixed $\lambda$. When the data are plotted against the radial distance from the point force scaled by system size, we find an excellent collapse in the intermediate regime and in the putative boundary layer. For the smallest system size considered, $R=L$, we note poorer data collapse in the near field region suggesting that one must study systems that are are least larger than a single filament length to access the bulk behavior of the network with quantitative accuracy. Clearly, all three curves taken together are consistent with the notion of an elastic boundary layer that is produced by the freely-rotating boundary at the wall and that extends distance approximately equal to $\lambda$ into the sample.

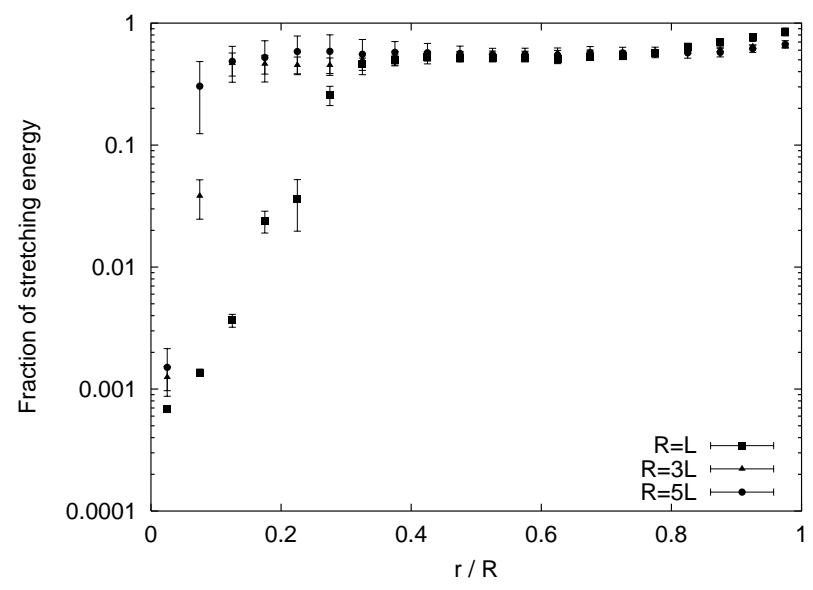

FIG. 14: The fraction of elastic energy stored in the $\mathcal{H}^{\|}$term of the Hamiltonian for perpendicular forces, as a function of distance from the origin $r$ for system sizes $R=L, 3 L$ and $5 L$. In all cases $L / l_{\mathrm{c}} \approx 29.1$ and $\lambda \approx 0.191 L$.

\section{B. Network response to force dipoles}

We now consider the mechanical response of the network to localized force dipoles at the origin. Understanding this response function is central to elucidating the effect of molecular motor activity in the cytoskeleton. Many of the general features observed in the response of the system to point force monopoles are also in evidence. For example in Fig. 15] we see the rough collapse in the far field of the mode amplitude $h_{0}^{r}(r)$ to the continuum solution for three different networks having the same value of $\lambda$. Note that the amplitude has been scaled by $l_{\mathrm{c}}^{2}$ since the magnitude of the imposed force dipole for each realization of the network will depend on the distance to the next constraint, i.e. cross-link, which is $l_{\mathrm{c}}$. Since the displacement field when averaged over network realizations is quadrupolar, being the difference in two dipole, that length must enter squared. The analogous displacement field amplitude, $h_{0}^{r}(r)$ from the continuum solution (solid line in Fig. 15) was calculated using unit forces so this amplitude is known only up to an overall scale factor; that scale factor was adjusted to best fit the data. A similar comparison can be made for the other continuum modes of the displacement field (see the Appendix). Fig. 16] for example, shows $h_{0}^{f}(r)$ with the same arbitrary prefactor. The agreement to the continuum theory scaled as discussed above is quite poor. As is also seen in the point-force response of the strain field, higher angular modes, which vanish in the continuum theory such as $h_{4}^{f}$ are significantly non-zero in the data. The amplitude of the non-continuum modes in the dipolar response is even more dramatic than in the point-force response of the network examined earlier.

The simulation data for the response of the network to force dipoles can be broadly summarized as follows: The amplitude of the continuum modes of the dipolar displacement field from the naive continuum theory do not agree with simulation data. In light of the discussion regarding such disagreements between the monopole data and the continuum calculation, this is perhaps not surprising. More interestingly, the observed displacement field has significant amplitudes of higher order angular modes. In short the universality of the response of semiflexible networks to localized point forces does not appear to extend to their response to localized force dipoles.

We speculate that the principal difference between these two cases stems from the fact that the network's response to the force dipole probes the more detailed microscopic structure of the network in the immediate vicinity of the point of the force dipole application. The amplitude of the lowest order force multipole communicated from the near field region to the far field where we expect a continuum based theory to apply is not constrained by elementary force balance. Neither are the amplitudes of any higher order force multipoles generated within the near field region. Thus, upon reaching the inner edge of the far field region the force dipole imposed at the origin has generated a highly complex set of tractions on the rest of the material whose structure depends on local details of the connectivity of the network near the origin. In contrast for the case of the force monopole, the dominant term in those tractions is the fixed total monopolar force acting on the intermediate region. The higher order force 
multipoles created in the inner region decay rapidly with distance from the origin leaving one with highly reproducible results for the response of the network to applied forces. The amplitude of the force dipole communicated from the near field region to the intermediate region, on the other hand, is not similarly constrained. It appears that one generically generates large amplitude higher order force multipoles in addition to whatever force dipole is communicated to the intermediate region making convergence to a simple dipolar form slow and difficult to observe in our finite samples.

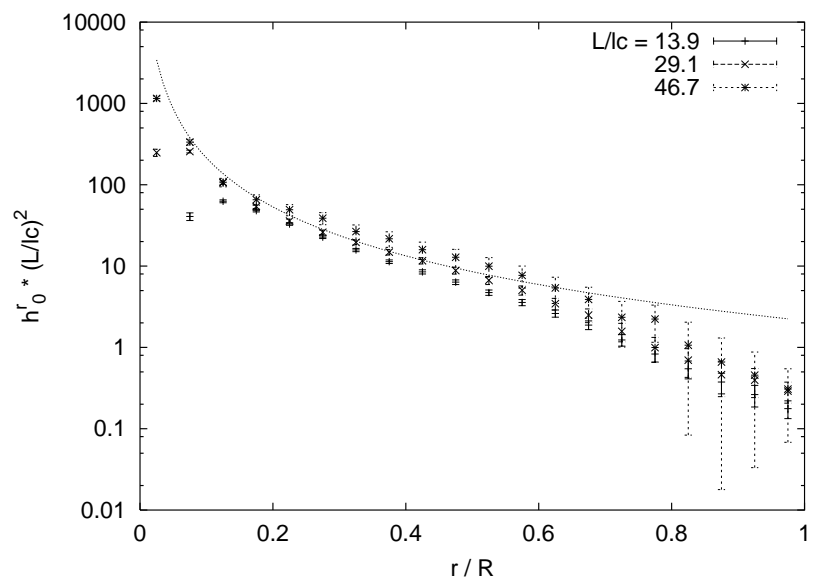

FIG. 15: $h_{0}^{r}$ for dipole forcing for fixed $\lambda / L \approx 0.191$ compared to the continuum solution (smooth line), which has one free fitting parameter, namely the overall magnitude of the dipole forcing. Both data and curve are negative, so the magnitudes have been plotted to allow use of a logarithmic axis. Each data curve has been scaled by $\left(L / l_{\mathrm{c}}\right)^{2}$ to ensure the same mean dipole magnitude (see text). The system radius was $R=L$.

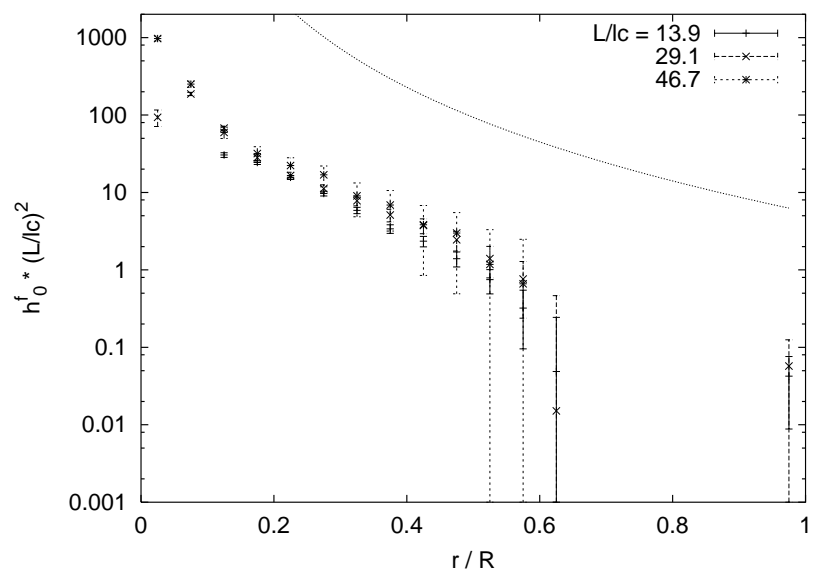

FIG. 16: $h_{0}^{f}$ for same systems as in Fig. 15] The only fitting parameter for the continuum solution is the same as used previously for $h_{0}^{r}$, so there are no remaining free fitting parameters in this plot. Much of the data for $r / R>0.6$ is negative and hence not visible on these axes.

\section{Bulk moduli}

Lastly, we present new results on homogeneous deformations of semiflexible networks. It has already been shown that the macroscopic elastic moduli of this class of model networks depend in a crucial way on the ratio of $\lambda$ to the filament length $L[5,8]$. For $\lambda / L \ll 1$, the deformation is approximately affine, whereas non-affine deformation modes dominate when $\lambda / L \gtrsim 1$. Previously this was demonstrated only for the shear modulus; here we can now confirm that the Young's modulus $Y$ behaves in an identical manner. Fig. [17] shows $Y$ measured from uniaxial extension of a rectangular cell, scaled by the prediction for an affine strain, plotted against $\lambda / L$ for the range of $L / l_{\mathrm{c}}$ considered in this paper. There is a clear data collapse, as for the shear modulus. Furthermore the deviation from the affine prediction is small for $\lambda / L \ll 1$, but becomes increasingly pronounced as $\lambda / L$ increases. This confirms that $\lambda / L$ controls the macroscopic elastic response of these systems.

Fig. 18 gives the Poisson ratio $\nu$ for the same systems as in Fig. 177 It is striking to observe that, within error bars, $\nu$ is consistent with the value $\nu=\frac{1}{2}$, which is the value expected for an affine deformation [8]. However, it is apparent from this figure that the measured values are consistent with $\nu=\frac{1}{2}$ for all data points, even those well into the non-affine regime. The mechanism behind this striking robustness currently evades us (is has nothing to do with incompressibility, which fixes $\nu=1$ in two dimensions). Note that $\nu$ at the rigidity percolation $L / l_{\mathrm{c}} \approx 5.933$ (at which the elastic moduli vanish) is $\approx \frac{1}{3}$ [7], which is clearly inconsistent with the data in Fig. 18 and confirms our earlier claims that the nonaffine regime is distinct from the scaling regime of the transition.

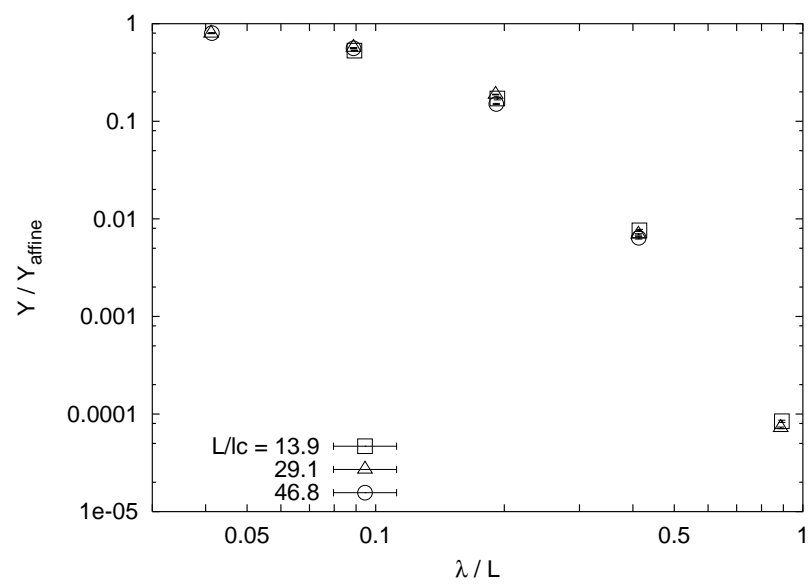

FIG. 17: Young's moduli scaled by the affine prediction versus $\lambda / L$ on $\log -\log$ axes. The affine prediction, which depends only on $L / l_{\mathrm{c}}$, can be found in [8]. The symbols are larger than the error bars. 


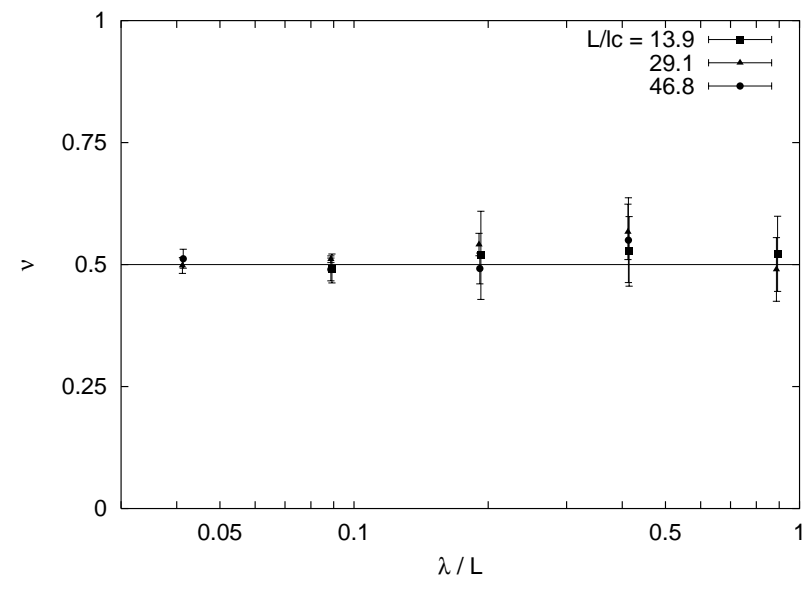

FIG. 18: The Poisson ratio $\nu$ for the same systems as in Fig. 17] The solid line corresponds to $\nu=\frac{1}{2}$.

\section{DISCUSSION}

In this paper we have presented the results of numerical studies on the response of semiflexible networks to both point forces and to homogeneously imposed strain. The data presented on the Young's modulus taken in combination with previous work on the static shear modulus shows that the mechanical response of the system can be understood in terms of Lamé coefficients that depend on the ratio of the filament length to the nonaffinity length: $L / \lambda$. The Poisson ratio of the material, however, appears to be remarkably insensitive to this ratio. We can offer no explanation for this insensitivity at this time. The data presented on the point force response form a necessary compliment to previous work on the development of a long length scale elastic theory of such materials.

Based on these data, it appears that the storage of elastic energy and the structure of the strain field is rather complex in the immediate vicinity of the applied point force. We characterize these quantities by considering three qualitatively different regimes as a function of radial distance from the applied point force. Immediately surrounding the point force in the near-field regime we find that the partitioning of elastic energy into bending and stretching is determined primarily by the angle between the applied force and the direction of the filament to which that force is applied. The disorder-averaged strain field is rather complex having higher angular harmonics that predicted by continuum elasticity theory. In the intermediate field regime farther from the point force the partitioning of elastic energy is determined solely by the ratio $L / \lambda$ as found in the homogeneous shear measurements. The higher angular harmonics present in the strain field appear to decay exponentially with a decay length proportional to $\lambda$ with a constant of proportionality near unity. These results taken in combination with our previous work suggests that one may think of $\lambda$ as setting the minimum length scale for the applicability of continuum modeling quite generally.

In the quasi-continuum regime $(r>\lambda)$, we find a strain field consistent with structure of that predicted by continuum model. The strain itself, however, cannot be simply computed from a knowledge of the effective Lamé coefficients. We believe the source of this discrepancy is the fact that the intermediate region, being poorly described by the continuum theory, applies a much more complex set of tractions to the material in the far-field where the continuum theory must apply. If these boundary conditions were known, we suspect that one could in fact calculate the resulting displacement field using the continuum theory. This belief is supported by the fact that in the far-field regime the displacement field for different networks having the same $\lambda$ collapse onto the same curve as one would expect for any effective continuum theory in which the Lamé coefficients depend on $\lambda$.

To summarize these results, we suggest that there is an emerging description of the mechanics semiflexible networks. There mechanical behavior over length scales longer than $\lambda$ appears to be described by a modified elastic theory in which the effective Lamé constants depend on the ratio $L / \lambda$. For point forces and presumably any force applied over regions having a characteristic length scale that is small compared to $\lambda$, the local response of the network is quite complex and the material appears to be anomalously compliant; at longer length scales, however, the structure of the deformation field appears to be consistent with the predictions of continuum elasticity. We believe that it should be possible to construct an elastic continuum theory of these networks that is applicable in both the intermediate and far-field and that is based on a modified gradient expansion of the strain field incorporating explicitly the mesoscopic length $\lambda$. The behavior of the strain field on scales much smaller than $\lambda$ appears to depend on other, more microscopic length scales.

Understanding the response of semiflexible networks to localized forces is a necessary for both microrheological investigations of semiflexible networks and for understanding the effect of molecular motors in the cytoskeleton. Clearly, further numerical investigations are required as well as a theoretical examination of the development of elastic continuum models applicable to the intermediate and far-field regimes.

Moreover, additional investigations are required to examine the analogous questions in three dimensional semiflexible networks. While we expect the basic physics outlined above, including the existence of a mesoscopic length $\lambda$, to persist in three dimensions, the expected $1 / r$ decay of the elastic Green's function should alter the results. Moreover the more rapid decay of the displacement field and energy density in the continuum three dimensional system may further simplify the structure of the analogous Green's functions for the semiflexible network. 


\section{Acknowledgements}

AJL and FCM acknowledge the hospitality of the Isaac Newton Institute for Mathematical Sciences where part of this work was done. DAH was jointly funded by the European Community Marie Curie and the Japanese Society for the Promotion of Science programs. AJL was supported in part by nsf-dmr0354113

\section{APPENDIX}

Here we derive the displacement field $\mathbf{u}(\mathbf{x})$ predicted by continuum elasticity for the cases of both monopole and dipole forcing. For the monopole case, a point force $\mathbf{f} \delta(\mathbf{x})$ is applied to the origin of an elastic sheet with Lamé coefficients $\mu$ and $\lambda$, or equivalently $\mu$ and the Poisson ratio $\nu=\lambda /(2 \mu+\lambda)$. For an isotropic elastic body, the stress obeys $\sigma_{i j}=\mu\left(\partial_{i} u_{j}+\partial_{j} u_{i}\right)+\lambda \delta_{i j} \nabla \cdot \mathbf{u}$ and the resulting equation for force balance is [21]

$$
(\mu+\lambda) \partial_{i}(\boldsymbol{\nabla} \cdot \mathbf{u})+\mu \nabla^{2} u_{i}=f_{i} \delta(\mathbf{x}) .
$$

Solving this in polar coordinates $(r, \phi)$ with the boundary condition $\mathbf{u} \equiv \mathbf{0}$ at a radius $R$ from the origin eventually leads to

$$
\begin{aligned}
u_{i}^{\text {mono }}= & \frac{f}{8 \pi \mu}\left\{4 \hat{f}_{i} \ln (r / R)-4 c_{1} \hat{r}_{i} \hat{r}_{j} \hat{f}_{j}\right. \\
& +c_{2}(r / R)^{-2}\left[2 \hat{r}_{i} \hat{r}_{j} \hat{f}_{j}-\hat{f}_{i}\right] \\
& \left.-(r / R)^{2}\left[2 \hat{r}_{i} \hat{r}_{j} \hat{f}_{j}-c_{2} \hat{f}_{i}\right]\right\},
\end{aligned}
$$

where $c_{1}=(1+\nu) /(3-\nu)$ and $c_{2}=(5+\nu) /(3-\nu)$. Then the only two non-zero modes according to the definition of the $g^{(\cdot)}$ given in (4) are

$$
\begin{aligned}
& g_{0}^{(r)}=\frac{\mu_{\mathrm{aff}}}{8 \pi \mu}\left\{-4 c_{1}+2 c_{2}(r / R)^{-2}-2(r / R)^{2}\right\}, \\
& g_{0}^{(f)}=\frac{\mu_{\mathrm{aff}}}{8 \pi \mu}\left\{4 \ln (r / R)-c_{2}(r / R)^{-2}+c_{2}(r / R)^{2}\right\} .
\end{aligned}
$$

A naive calculation of the corresponding dipole solution would simply superpose the above monopole solution for two point forces, $\mathbf{f} \delta(\mathbf{x})$ and $-\mathbf{f} \delta(\mathbf{x}-\boldsymbol{\varepsilon})$ with $\boldsymbol{\varepsilon}=\varepsilon \hat{\mathbf{f}}$, and then take the limit $\varepsilon \rightarrow 0$. However, this ignores the boundary at radius $R$, which should be kept fixed but is shifted a distance $\varepsilon$ by the above procedure. An exact calculation would require the monopole solution for force applied near to (but not at) the center of a circular system, which, since it no longer obeys radial symmetry, is likely to be highly complex. Here we ignore such issues and simply use the above monopole solution, in the expectation that it will closely approximate the exact case except possibly near the boundary. The displacement field induced by the force dipole is then

$$
\begin{aligned}
& u_{i}^{\text {dip }}=-\frac{1}{\varepsilon} \nabla u_{i}^{\text {mono }} \cdot \varepsilon \\
& =\frac{f}{8 \pi \mu} \frac{1}{r}\left\{\hat{r}_{i}\left[4 c_{1}-2 c_{2}(r / R)^{-2}+2(r / R)^{2}\right]\right. \\
& +8 \hat{r}_{i}(\hat{\mathbf{r}} \cdot \hat{\mathbf{f}})^{2}\left[c_{2}(r / R)^{-2}-c_{1}\right] \\
& +2 \hat{f}_{i}(\hat{\mathbf{r}} \cdot \hat{\mathbf{f}})\left[2\left(c_{1}-1\right)-2 c_{2}(r / R)^{-2}\right. \\
& \left.\left.-\left(c_{2}-1\right)(r / R)^{2}\right]\right\} \text {. }
\end{aligned}
$$

As explained in Sec. IIC the dipole solution above applies for a single realization on large length scales, but after averaging over many networks on short lengths (as in the simulations) the dipole moment vanishes, leaving a quadrupole displacement field. The required quadrupole is one consisting of two parallel dipoles of equal and opposite magnitude, aligned along their axes, i.e.

$$
\begin{aligned}
u_{i}^{\text {quad }}= & \frac{1}{\varepsilon} \nabla u_{i}^{\text {dip }} \cdot \varepsilon \\
= & \frac{f}{8 \pi \mu} \frac{1}{r^{2}}\left\{\hat { f } _ { i } \left[4\left(2 c_{1}-1\right)-6 c_{2}(r / R)^{-2}\right.\right. \\
& \left.\quad+2\left(2-c_{2}\right)(r / R)^{2}\right] \\
& +24 \hat{r}_{i}(\hat{\mathbf{r}} \cdot \hat{\mathbf{f}})\left[c_{2}(r / R)^{-2}-c_{1}\right] \\
& +8 \hat{f}_{i}(\hat{\mathbf{r}} \cdot \hat{\mathbf{f}})^{2}\left[1-2 c_{1}+3 c_{2}(r / R)^{-2}\right] \\
& \left.+16 \hat{r}_{i}(\hat{\mathbf{r}} \cdot \hat{\mathbf{f}})^{3}\left[2 c_{1}-3 c_{2}(r / R)^{-2}\right]\right\} .
\end{aligned}
$$

This gives the displacement field in response to known forces of magnitude $f$. Since it is rather the displacement that is controlled, $f$ is a free parameter. Finally, the nonzero modes are

$$
\begin{aligned}
h_{0}^{(r)}= & \frac{\mu_{\text {affine }}}{\pi \mu} \frac{1}{r^{2}}\left\{-c_{1}\right\}, \\
h_{2}^{(r)}= & \frac{\mu_{\text {affine }}}{2 \pi \mu} \frac{1}{r^{2}}\left\{2 c_{1}-3 c_{2}(r / R)^{-2}\right\}, \\
h_{0}^{(f)}=\frac{\mu_{\text {affine }}}{4 \pi \mu} \frac{1}{r^{2}}\left\{3 c_{2}(r / R)^{-2}\right. & \left.+\left(2-c_{2}\right)(r / R)^{2}\right\}, \\
h_{2}^{(f)}= & \frac{\mu_{\text {affine }}}{4 \pi \mu} \frac{1}{r^{2}}\left\{1-2 c_{1}+3 c_{2}(r / R)^{-2}\right\} .
\end{aligned}
$$

Cell Biol. 3, 4 (1991).

[1] B. Alberts, D. Bray, J. Lewis, M. Raff, K. Roberts, and J.D. Watson, Molecular Biology of the Cell, 3rd edition (Garland, New York, 1994); P.A. Janmey Curr. Opin.
[2] T.D. Pollard and J.A. Cooper, Ann. Rev. Biochem. 55 987 (1986). 
[3] E.L. Elson Annu. Rev. Biophys. Biochem. 17, 397 (1988).

[4] M. Rubenstein and R.H. Colby, Polymer Physics (Oxford University Press, London, 2003).

[5] D.A. Head, A.J. Levine and F.C. MacKintosh, Phys. Rev. Lett 91, 108102 (2003).

[6] J. Wilhelm and E. Frey, Phys. Rev. Lett. 91, 108103 (2003).

[7] D.A. Head, F.C. MacKintosh and A.J. Levine, Phys. Rev. E 68, 025101(R) (2003).

[8] D.A. Head, A.J. Levine and F.C. MacKintosh, Phys. Rev. E 68, 061907 (2003).

[9] Alex J. Levine, D.A. Head, and F.C. MacKintosh J. Phys.: Condens. Mat. 16, S2079 (2004).

[10] K. Kroy and E. Frey, Phys. Rev. Lett. 77, 306 (1996).

[11] R. Satcher and C. Dewey, Biophys. J. 71109 (1996).

[12] F.C. MacKintosh, J. Käs and P.A. Janmey, Phys. Rev. Lett. 75, 4425 (1995).

[13] J.P. Wittmer, A. Tanguy, J.-L. Barrat and L. Lewis, Europhys. Lett. 57, 423 (2002).
[14] A. Tanguy, J.P. Wittmer, F. Leonforte and J.-L. Barrat, Phys. Rev. B 66, 174205 (2002).

[15] F. Leonforte, A. Tanguy, J.P. Wittmer and J.-L. Barrat, Phys. Rev. B 70, 014203 (2004).

[16] C. Goldenberg and I. Goldhirsch, Phys. Rev. Lett. 89, 084302 (2002).

[17] C. Bustamante, J.F. Marko, E.D. Siggia, and S. Smith, Science 265, 1599 (1994).

[18] W.H. Press, S.A. Teukolsky, W.T. Vetterling and B.P. Flannery, Numerical recipies in $C$, 2nd ed. (CUP, Cambridge, 1992).

[19] P.R. Onck, T. Koeman, T. van Dillen and E. van der Giessen, cond-mat/0502397

[20] D. Bray, Cell Movements: From molecules to motility, 2nd ed. (Garland, New York, 2001).

[21] L.D. Landau and E.M. Lifshitz, Theory of elasticity, 3rd ed. (Reed, Oxford, 1986). 